\title{
Oxygen-Induced Conformational Changes in the PAS-Heme Domain of the Pseudomonas aeruginosa Aer2 Receptor
}

\section{Supporting Information (Table S1 and Figures S1-S5)}

Emilie Orillard, Selina Anaya, Mark S. Johnson, and Kylie J. Watts ${ }^{*}$

Division of Microbiology and Molecular Genetics, Loma Linda University, Loma Linda, CA, 92350, USA 
Table S1. Aer2 PAS sidechain solvent accessible surface area (SASA)

\begin{tabular}{|c|c|c|c|}
\hline Residue & PAS chain B $(\AA)^{a}$ & PAS chain D $(\AA)^{a}$ & Purpose \\
\hline 1175 & 18.43 & 13.25 & \multirow{14}{*}{ Experimental } \\
\hline L179 & 10.99 & 18.56 & \\
\hline V182 & 0.04 & 0 & \\
\hline S183 & 8.82 & 11.92 & \\
\hline A184 & 0 & 0 & \\
\hline V186 & 1.87 & 3.63 & \\
\hline D265 & 1.8 & 0.27 & \\
\hline V267 & 8.65 & 6.39 & \\
\hline A280 & 4.87 & 9.06 & \\
\hline Q282 & 9.75 & 10.3 & \\
\hline T284 & 33.32 & 32.51 & \\
\hline D285 & 17.88 & 17.59 & \\
\hline R286 & 79.04 & 85.22 & \\
\hline T287 & 30.29 & 24.79 & \\
\hline A189 & 0 & 0 & \multirow{4}{*}{$\begin{array}{l}\text { Negative } \\
\text { PEGylation and } \\
\text { cross-linking } \\
\text { controls }\end{array}$} \\
\hline L255 & 5.43 & 8.22 & \\
\hline S279 & 1.31 & 1.63 & \\
\hline V281 & 0 & 0 & \\
\hline R224 & 166.55 & 138.65 & \multirow{2}{*}{$\begin{array}{c}\text { Positive } \\
\text { PEGylation } \\
\text { controls }\end{array}$} \\
\hline $\mathrm{H} 242$ & 124.61 & 122.97 & \\
\hline
\end{tabular}

aUsing a $1.4 \AA$ radius and the $B$ and D PAS chains from PDB 4HI4. SASA was determined using the ProtSA server. Since the PAS dimer structure does not contain a C-terminally connected HAMP domain, the SASAs measured for the most C-terminal residues may not be accurate. 
Polyethylene glycol (PEG)maleimide

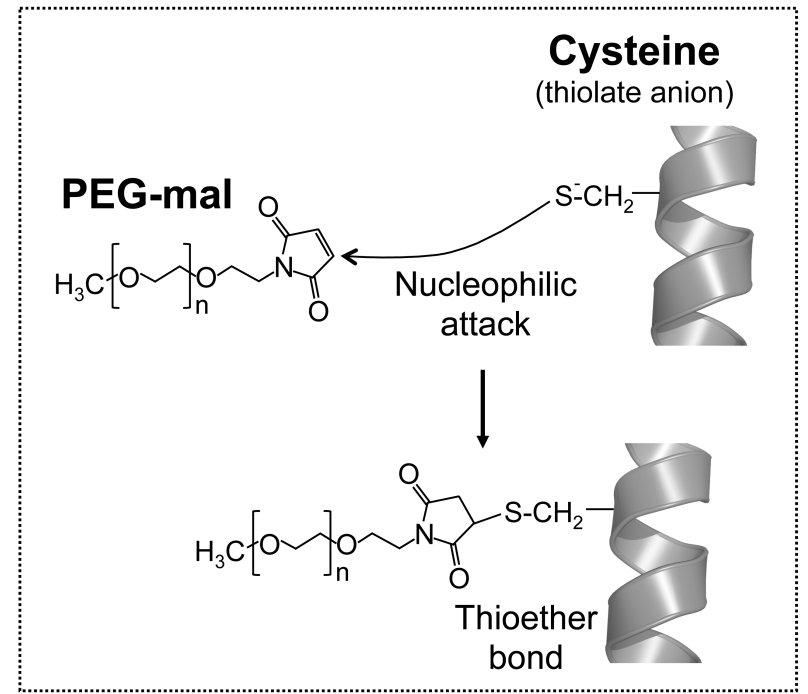

\section{Bismaleimidoethane (BMOE)}

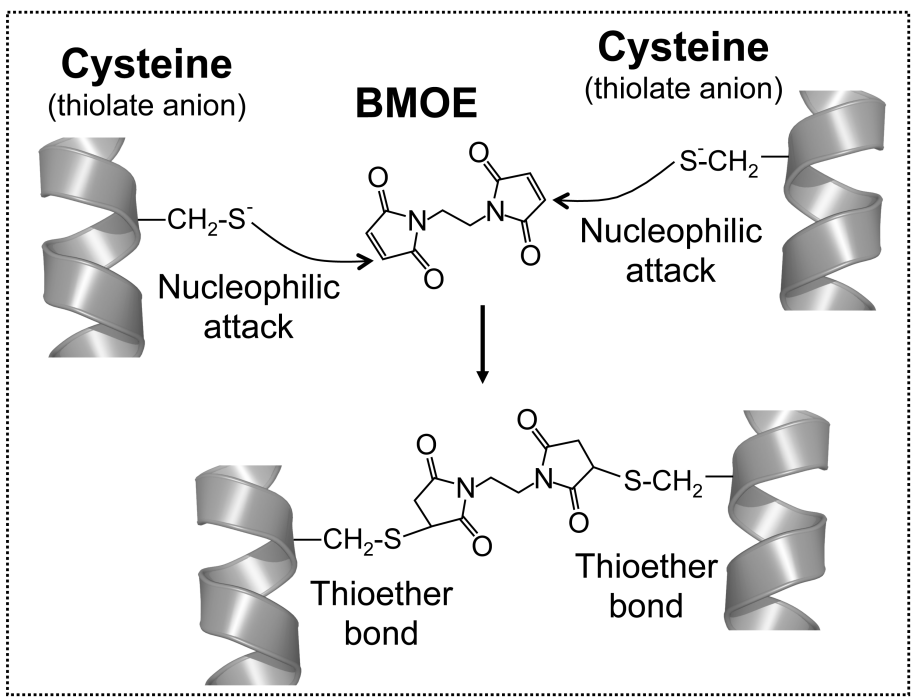

Figure S1. Structures and chemical reactions for polyethylene glycol (PEG)-maleimide and bismaleimidoethane (BMOE). The reaction of a thiolate anion with a maleimide group results in the formation of a stable thioether linkage. 


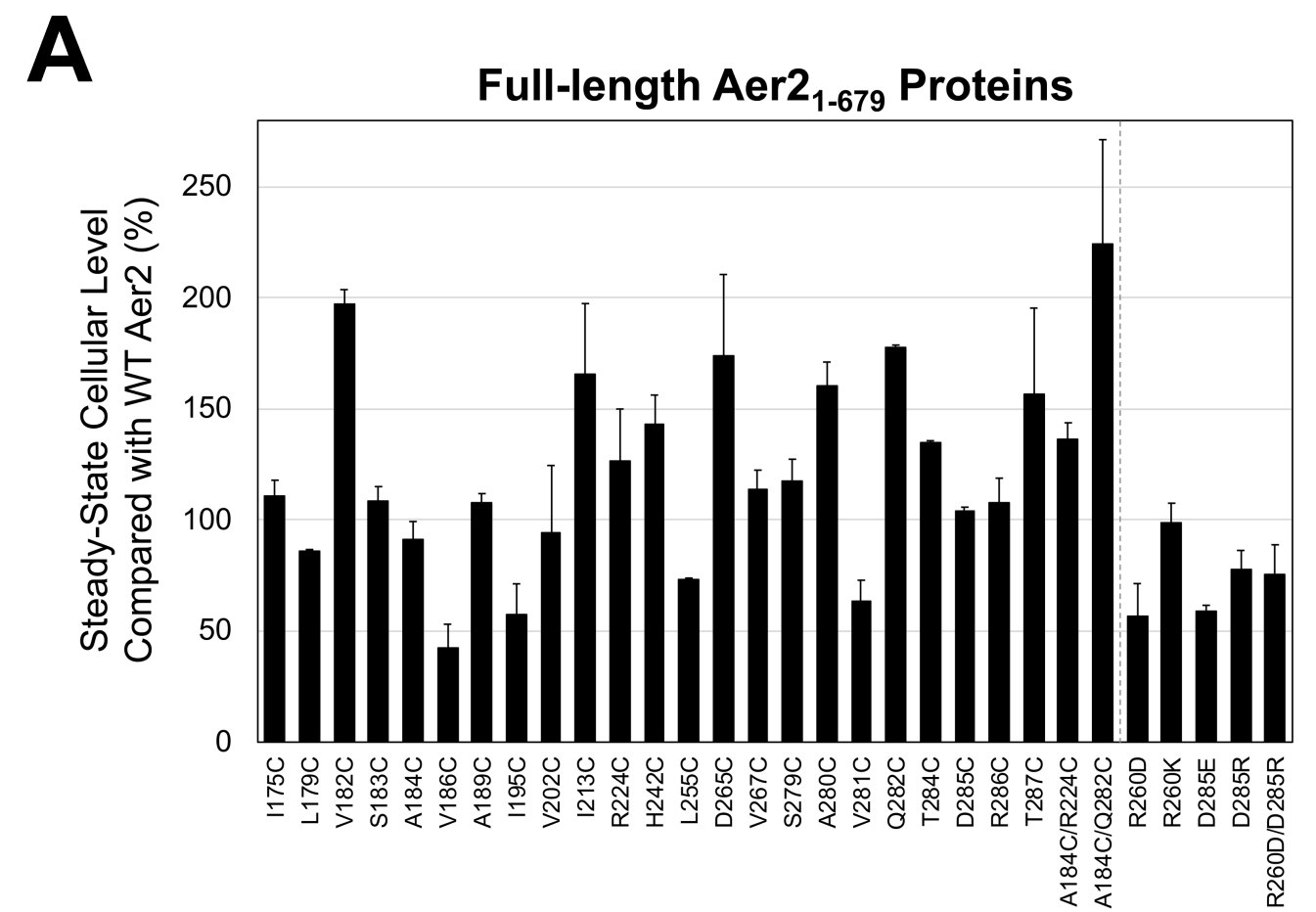

B
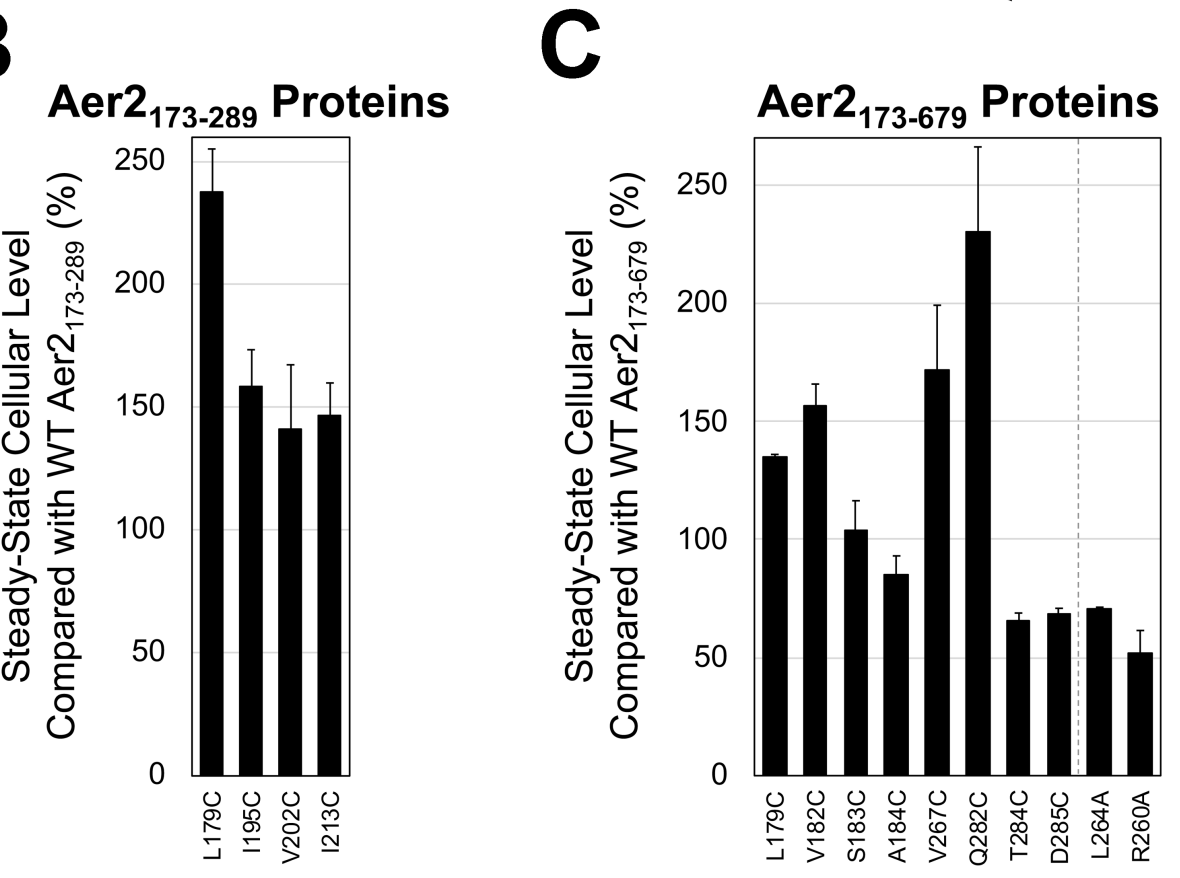

Figure S2. Steady-state cellular levels of the Aer2 proteins used in this study. (A) Steadystate levels of full-length Aer2 receptors (Aer2 ${ }_{1-679)}$ compared with WT Aer2 after inducing $E$. coli BT3388 with 50 MM IPTG. (B) Steady-state levels of PAS-Cys proteins (Aer2 173-289) compared with WT PAS after inducing E. coli BT3388 with $100 \mu \mathrm{M}$ IPTG. (C) Steady-state levels of Aer2 proteins lacking N-terminal HAMP 1-3 (Aer2 ${ }_{173-679)}$ compared with WT Aer2 ${ }_{173-}$ 679 after inducing $E$. coli BT3388 with $50 \mu \mathrm{M}$ IPTG. In all cases, error bars represent standard deviation from multiple experiments. 

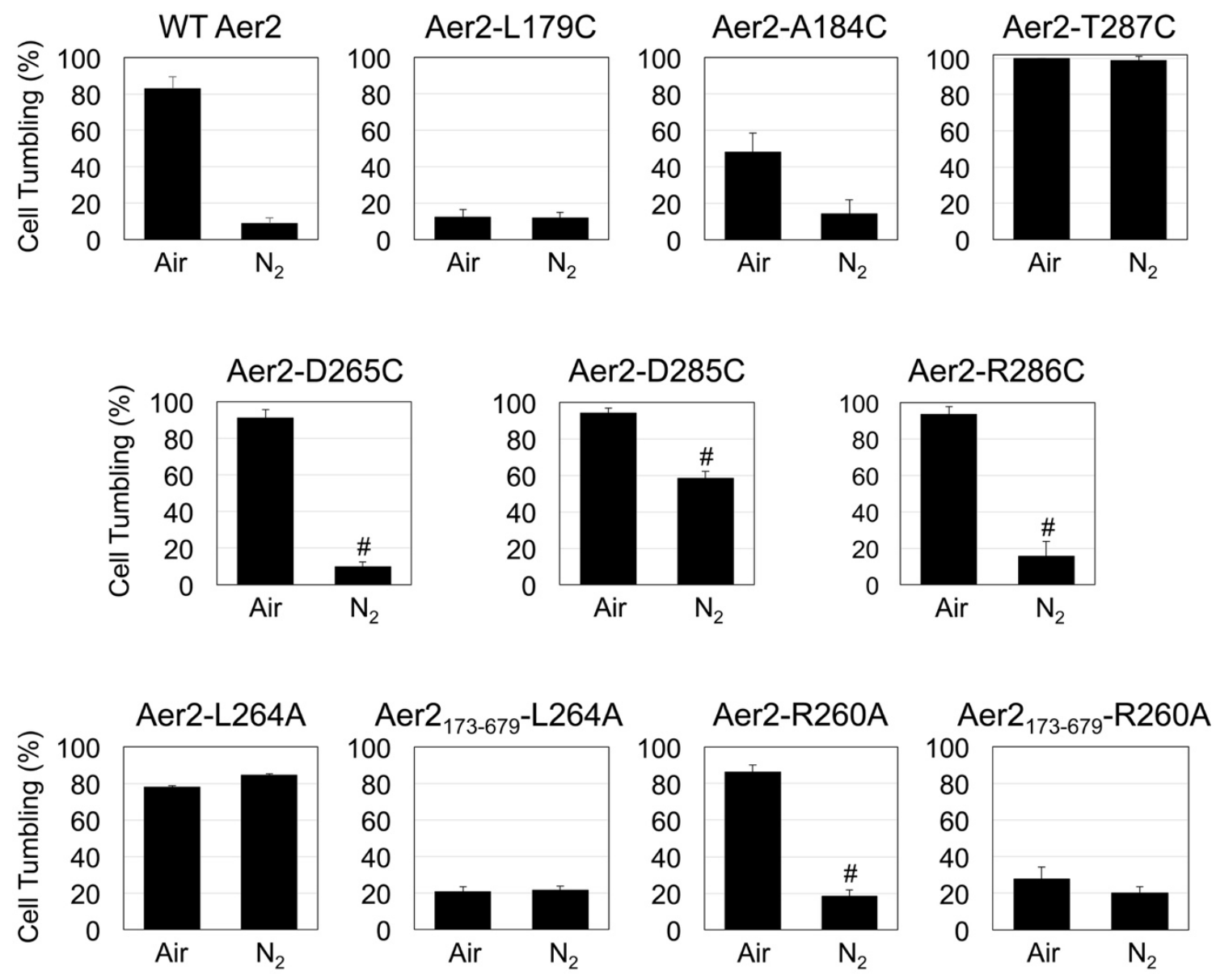

Figure S3. Example behavioral assays showing the average percent of $E$. coli $\mathrm{BT} 3388$ cells tumbling over a $1 \mathrm{~s}$ period $30 \mathrm{~s}$ after switching to air or $\mathrm{N}_{2}$. In addition to WT Aer2, the following Aer2 mutants are shown: a representative signal-off mutant (Aer2-L179C), signal-off biased mutants (Aer2-A184C, Aer2 173-679-L264A, and Aer2 173-679-R260A), signal-on mutants (Aer2-T287C and Aer2-L264A), and signal-on biased mutants (Aer2-D265C, Aer2-D285C, Aer2-R286C, and Aer2-R260A). Hash-tags indicate that smooth-swimming was delayed 10$20 \mathrm{~s}$ in $\mathrm{N}_{2}$ compared to WT Aer2. 


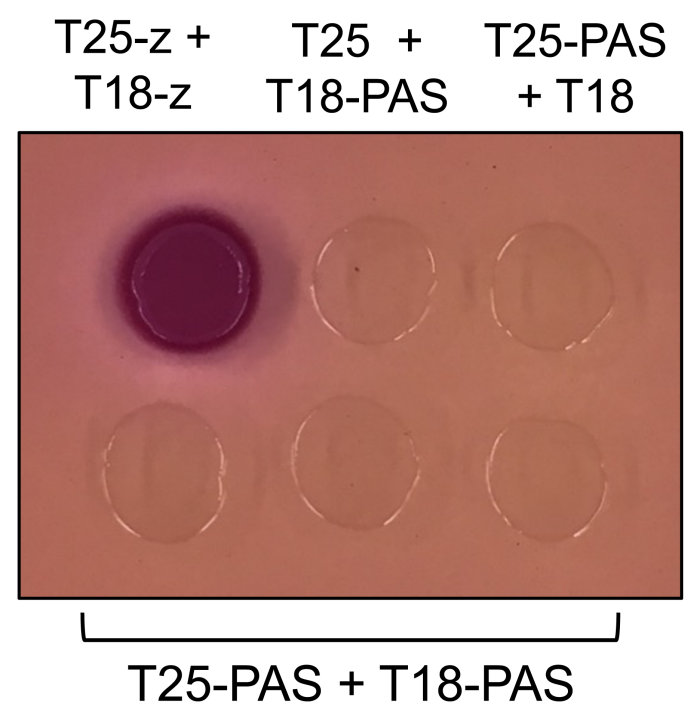

Figure S4. Lack of PAS-PAS interaction in BACTH assays after spotting E. coli BTH101 cells onto MacConkey agar and incubating for $48 \mathrm{~h}$ at $30^{\circ} \mathrm{C}$ (a representative photo is shown after $24 \mathrm{~h}$ of growth; results were the same at $48 \mathrm{~h})$. A red spot for the control (T25-z + T18-z, leucine zipper) indicates protein interaction, whereas colorless spots for the different combinations of empty vectors and T18-PAS (T18-Aer2 ${ }_{173-289}$ ) or T25-PAS (T25-Aer2 $173-289$ ) indicates no interaction. 


\section{Comparison of Aer2 ${ }_{1-679}$ and Aer2 ${ }_{173-679}$ Cross-linking with BMOE}

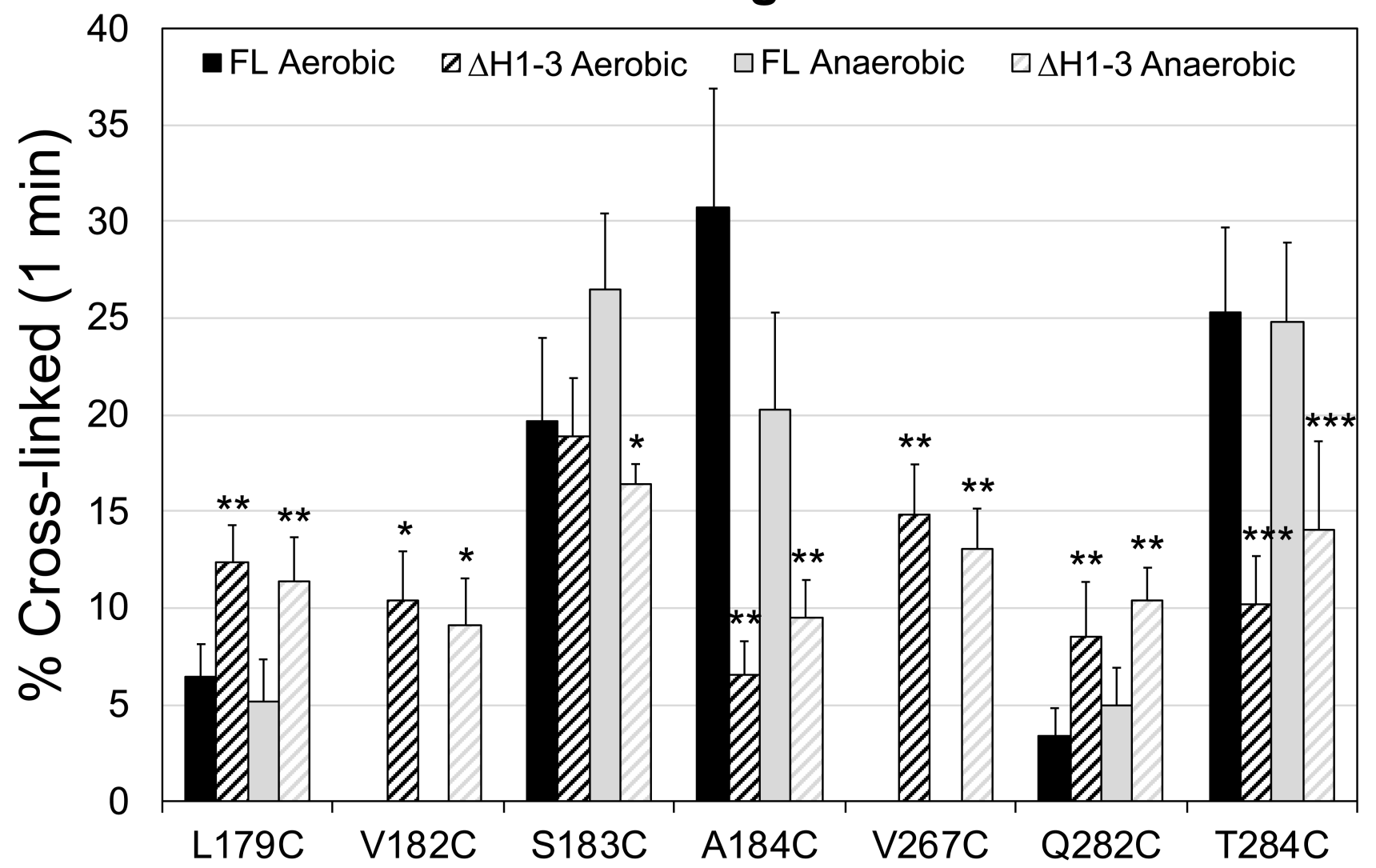

Figure S5. Comparison of average cross-linking extents for Aer21-679-Cys (full-length; FL) and

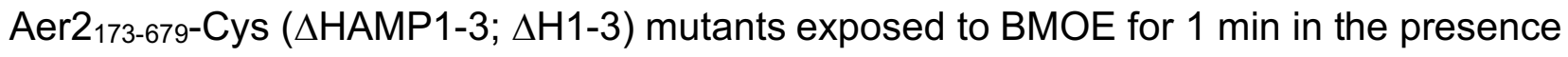
(black bars) and absence (gray bars) of $\mathrm{O}_{2}$. Error bars represent standard deviation from multiple experiments. ${ }^{*} P<0.05 ;{ }^{* *} P<0.01 ;{ }^{* * *} P<0.001$. 